\title{
On constructions of hypotraceable graphs
}

\author{
Gábor Wiener ${ }^{1,2}$ \\ Department of Computer Science and Information Theory \\ Budapest University of Technology and Economics \\ Budapest, Hungary
}

\begin{abstract}
A graph $G$ is hypohamiltonian/hypotraceable if it is not hamiltonian/traceable, but all vertex deleted subgraphs of $G$ are hamiltonian/traceable. Until now all hypotraceable graphs were constructed using hypohamiltonian graphs; extending a method of Thomassen [8] we present a construction that uses so-called almost hypohamiltonian graphs (nonhamiltonian graphs, whose vertex deleted subgraphs are hamiltonian with exactly one exception). As an application, we construct a planar hypotraceable graph of order 138, improving the best known bound of 154 [5]. We also prove a structural type theorem showing that hypotraceable graphs possessing some connectivity properties are all built using either Thomassen's or our method.
\end{abstract}

Keywords: hypotraceable graph, hypohamiltonian graph, almost hypohamiltonian graph

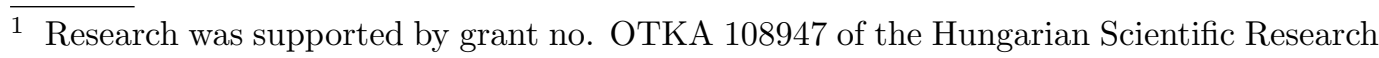
Fund and by the János Bolyai Research Scholarship of the Hungarian Academy of Sciences.

2 Email: wiener@cs.bme.hu 


\section{Introduction}

All graphs considered in this paper are finite, simple, and connected. For a graph $G, V(G)$ and $E(G)$ denotes the set of vertices and edges of $G$, respectively. The edge between vertices $a$ and $b$ is denoted by $(a, b)$. The subgraph of $G$ induced by the vertex set $X$ is denoted by $G[X]$ and $G-X:=G[V(G) \backslash X]$, $G-v:=G-\{v\}$ for any $v \in V(G)$ and for $e \in E(G)$, let $G-e$ denote the graph obtained by deleting $e$ from $E(G)$.

A graph $G$ is hypohamiltonian (hypotraceable) if $G$ is not hamiltonian (traceable), but for any vertex $v \in V(G), G-v$ is hamiltonian (traceable). $G$ is almost hypohamiltonian with exceptional vertex $w \in V(G)$ if $G$ and $G-w$ are not hamiltonian, but for any vertex $v$ of $V(G-w), G-v$ is hamiltonian. The study of hypohamiltonian graphs started in 1963 [7], but an example of such a graph is known for a much longer time: the Petersen graph is hypohamiltonian, actually it is the smallest hypohamiltonian graph [3]. Hypotraceable graphs are much harder to find, actually in 1968 Kapoor et al. [6] conjectured that such graphs do not exist. This was disproved in 1974 by Horton (see [10], [9]), who found a hypotraceable graph of order 40. His construction was generalized by Thomassen [9], who also found another method to create hypotraceable graphs [8]. The smallest known hypotraceable graph is constructed by this latter method and has 34 vertices. Almost hypohamiltonian graphs were introduced by C. T. Zamfirescu [11], the smallest known such graph has 17 vertices. It is worth mentioning that until 1976 all hypohamiltonian graphs known were non-planar (actually, of crossing number at least 2), which led Chvátal to ask whether planar hypohamiltonian graphs exist [1]. The first such graph (of order 105) was found by Thomassen [9], the smallest known planar hypohamiltonian, hypotraceable, and almost hypohamiltonian graphs have order 40 [5], 154 [5], and 39 [11], respectively. For more about hypohamiltonicity and hypotraceablity, see the survey paper by Holton and Sheehan [4]

The paper is organized as follows. In Section 2 we extend Thomassen's construction [8] so that it can use almost hypohamiltonian graphs instead of hypohamiltonian graphs. As an application, we construct a planar hypotraceable graph of order 138, improving the best known bound of 154 [8]. The graph is based on a new planar almost hypohamiltonian graph of order 36, which also has its own interest, being the smallest known almost hypohamiltonian graph with exceptional vertex of degree 3, improving the bound of 47 in [11] (concerning the importance of these graphs also see [11]), and also the smallest known planar almost hypohamiltonian graph, improving the bound 
of 39 also presented in [11]. In Section 3 we prove a structural type theorem: 2-fragments of hypotraceable graphs having edge connectivity 2 are built from two vertex deleted hypohamiltonian or almost hypohamiltonian graphs. That is, all hypotraceable 2 -fragments of edge connectivity 2 can be essentially built using Thomassen's construction [8] or the construction given in Section 2.

\section{Constructing hypotraceable graphs}

Definition 2.1 Let $G$ be a non-complete graph with connectivity $k$ and $X=$ $\left\{x_{1}, x_{2}, \ldots, x_{k}\right\}$ be a cut of $G$. Let furthermore $H$ be one of the components of $G-X$. Then $G[V(H) \cup X]$ is called a $k$-fragment of $G$, and $X$ is called the vertices of attachment of $H$.

Definition 2.2 Let $G$ be a graph, $v \in V(G)$. $G$ is said to be $v$-traceable if there exists a hamiltonian path of $G$ starting at $v$.

The following theorem, which characterizes the 2-fragments of hypotraceable graphs is due to Thomassen [9].

Theorem 2.3 (Thomassen, 1976) Let $G$ be a graph, such that $G=G_{1} \cup G_{2}$ and let $x, y \in V(G)$. Suppose furthermore that $V\left(G_{1}\right) \cap V\left(G_{2}\right)=\{x, y\}$ and that both $G_{1}$ and $G_{2}$ have at least 3 vertices. Then $G$ is hypotraceable if and only if for $i=1,2, G_{i}$ is not $x$-traceable and not $y$-traceable, but for any $v \in V\left(G_{i}\right), G_{i}-v$ is $x$-traceable or $y$-traceable.

A graph $H$ possessing the property that for some $x, y \in V(H), H$ is not $x$ traceable and not $y$-traceable, but for any $v \in V(H), H-v$ is $x$-traceable or $y$-traceable, will be simply called a hypotraceable 2 -fragment (with vertices of attachment $x, y$ ). Thomassen constructed hypotraceable 2-fragments from hypohamiltonian graphs in [8]; here we extend his method in order to create hypotraceable 2-fragments from almost hypohamiltonian graphs. (Actually, 2 -fragments of hypotraceable graphs are not mentioned in [8], Thomassen constructs the hypotraceable graphs of connectivity 2 directly - but the 2 fragments are used implicitly.)

Lemma 2.4 Let $G$ be an almost hypohamiltonian graph with exceptional vertex $y$ of degree 3 and suppose that all three neighbours $y_{1}, y_{2}, y_{3}$ of $y$ also have degree 3. Then there exist at least two among the $y_{i}$ 's for which $G-y$ is $y_{i}$-traceable.

The proof of the lemma is omitted due to space limitations. 
Let $G_{i}$ be an almost hypohamiltonian graph with exceptional vertex $x_{i}$ of degree 3 and suppose that all three neighbours $a_{i}, b_{i}, c_{i}$ of $x_{i}$ also have degree 3 for $i=1,2$. By Lemma 2.4, we may suppose w.l.o.g. that $G_{i}-x_{i}$ is $a_{i^{-}}$ traceable and $b_{i}$-traceable for $i=1,2$. Let now $G$ be the graph obtained from the disjoint union of $H_{1}:=G_{1}-x_{1}$ and $H_{2}:=G_{2}-x_{2}$ by adding the edges $\left(a_{1}, a_{2}\right)$ and $\left(b_{1}, b_{2}\right)$.

Theorem 2.5 The graph $G$ constructed above is a hypotraceable 2-fragment with vertices of attachment $c_{1}, c_{2}$.

Proof. First let us prove that $G$ is not $c_{i}$-traceable for $i=1,2$. Assume to the contrary that there exists a hamiltonian path $P$ of $G$ starting at (w.l.o.g. say) $c_{1}$. The other endvertex of $P$ cannot be in $H_{1}$, otherwise there would exist a hamiltonian path of $H_{2}$ between $a_{2}$ and $b_{2}$ and then $G_{2}$ would be hamiltonian. On the other hand, the other endvertex of $P$ cannot be in $H_{2}$ either, otherwise there would exist a hamiltonian path of $H_{1}$ between $c_{1}$ and either $a_{1}$ or $b_{1}$, and then $G_{1}$ would be hamiltonian.

Now we show that for any $v \in V(G), G-v$ is $c_{1}$-traceable or $c_{2}$-traceable. Let us assume w.l.o.g. that $v \in V\left(H_{2}\right)$. Since $G_{2}-v$ is hamiltonian, there is a hamiltonian path between two of the vertices $a_{2}, b_{2}, c_{2}$ in $G_{2}-v-x=H_{2}-v$. Now we distinguish two cases.

Case 1. There is a hamiltonian path $P$ between $a_{2}$ and $b_{2}$ in $H_{2}-v$. Since $G_{1}-b_{1}$ is hamiltonian, there is a hamiltonian path $Q$ of $G_{1}-b_{1}-x=H_{1}-b_{1}$ between $a_{1}$ and $c_{1}$. Now $\left(b_{1}, b_{2}\right) \cup P \cup\left(a_{1}, a_{2}\right) \cup Q$ is a hamiltonian path of $G-v$ between $b_{1}$ and $c_{1}$.

Case 2. There is a hamiltonian path $P$ between $c_{2}$ and either $a_{2}$ or $b_{2}$ in $H_{2}-v$. Let us suppose w.l.o.g. that $P$ is between $c_{2}$ and $a_{2}$. We know that $H_{1}$ is $a_{1}$-traceable; let $Q$ be a hamiltonian path of $H_{1}$ starting at $a_{1}$. Now $P \cup\left(a_{1}, a_{2}\right) \cup Q$ is a hamiltonian path of $G$ starting at $c_{2}$.

Now it is easy to create hypotraceable graphs from almost hypohamiltonian graphs: we just have to create the hypotraceable 2-fragments $H_{1}$ and $H_{2}$ with vertices of attachment $x_{1}, y_{1}$ and $x_{2}, y_{2}$, respectively and glue them together, that is, take their disjoint union and identify $x_{1}$ with $x_{2}$ and $y_{1}$ with $y_{2}$. It is worth mentioning that until now all known hypotraceable graphs were constructed based on one of the methods of Thomassen [8], [9] and that the construction here is the first one that does not use hypohamiltonian graphs as building blocks. In order to actually use the construction we need almost hypohamiltonian graphs, where the exceptional vertex and its neighbours have degree 3. C. T. Zamfirescu [11] shows the existence of a 47 vertex almost 
hypohamiltonian graph $G$ with an exceptional vertex $w$ of degree 3 and it is also true that all neighbours of $w$ have degree 3. C. T. Zamfirescu also proves that almost hypohamiltonian graphs with an exceptional vertex of degree 3 of order $n$ exist for every $n \geq 84$. The proof easily extends to the case when we also require that the neighbours of the exceptional vertex also have degree 3 . As it was mentioned in the Introduction, the smallest planar hypotraceable graph (which is based on four copies of the 40 vertex planar hypohamiltonian graph of Jooyandeh et al. [5]) is of order 154, and appears also in [5]. In order to improve on this, we present a 36 vertex planar almost hypohamiltonian graph $G$ (Figure 1) with exceptional vertex $w$ of degree 3 , such that the neighbours of $w$ also have degree 3 . Using $G$ as both $G_{1}$ and $G_{2}$ in our construction we obtain a planar hypotraceable 2-fragment of order 70 and therefore we obtain a planar hypotraceable graph of order 138.

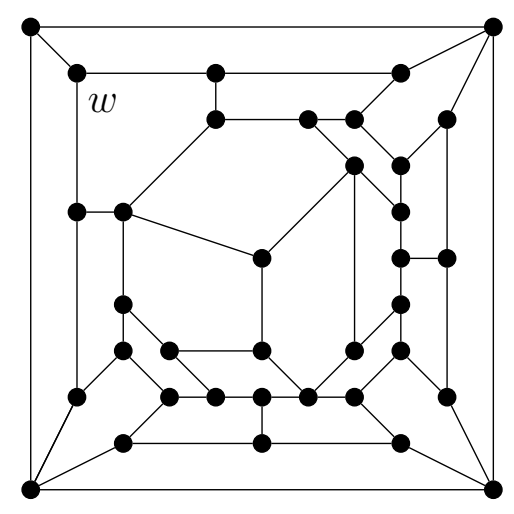

Fig. 1. An almost hypohamiltonian graph $G$ of order 36

Theorem 2.6 The graph $G$ of Figure 1 is almost hypohamiltonian with exceptional vertex $w$.

Proof. The nonhamiltonicity of $G$ and $G-w$ can be proved using Grinberg's theorem [2], the details are omitted here due to lack of space. The hamiltonicity of the vertex-deleted subgraphs of $G$ (except $G-w$ ) can be easily verified using mathematical softwares, like the open-source SageMath.

\section{Structure of hypotraceable graphs}

We have seen that we do not necessarily need hypohamiltonian graphs to construct hypotraceable graphs. However, all known hypotraceable graphs are still created from building blocks based on hypohamiltonian or almost hypohamiltonian graphs. It seems pretty natural to ask whether all hypotraceable 
graphs must contain such building blocks. The following theorem gives a partial answer to this question.

Theorem 3.1 Let $G$ be a hypotraceable 2-fragment of edge connectivity 2 and $\left\{e_{1}, e_{2}\right\}$ an edge cut of $G$. Let furthermore $G_{1}$ and $G_{2}$ be the components of $G-e_{1}-e_{2}$. Then $G_{1}$ and $G_{2}$ are vertex deleted hypohamiltonian or almost hypohamiltonian graphs.

The proof of the theorem is omitted here due to the page limit.

\section{References}

[1] V. Chvátal, Flip-flops in hypohamiltonian graphs, Can. Math. Bull. 16 (1973), $33-41$.

[2] E. J. Grinberg, Plane homogeneous graphs of degree three without Hamiltonian circuits, Latvian Math. Yearbook, Izdat. Zinatne, Riga 4 (1968), 51-58. (In Russian)

[3] J. C. Herz, T. Gaudin, P. Rossi, Solution du probléme No. 29, Revue Francaise de Recherces Opérationelle 8 (1964), pp. 214-218.

[4] D. A. Holton and J. Sheehan, Hypohamiltonian graphs, The Petersen Graph, Cambridge University Press, New York, 1993.

[5] M. Jooyandeh, B. D. McKay, P. R. J. Östergård, V. H. Pettersson, C. T. Zamfirescu, Planar Hypohamiltonian Graphs on 40 Vertices, J. Graph Theory DOI: $10.1002 /$ jgt.22015 (2016)

[6] S. F. Kapoor, H. V. Kronk, and D. R. Lick, On detours in graphs, Canad. Math. Bull. 11 (1968), 195-201.

[7] R. Sousselier, Probleme No. 29: Le Cercle des Irascibles, Revue Française de Recherches Operationelles 7 (1963), 405-406.

[8] C. Thomassen, Hypohamiltonian and hypotraceable graphs, Discrete Mathematics 9 (1974), 91-96.

[9] C. Thomassen, Planar and infinite hypohamiltonian and hypotraceable graphs, Discrete Mathematics 14 (1976), 377-389.

[10] T. Zamfirescu, On longest paths and circuits in graphs, Math. Scand. 38 (1976), $211-239$.

[11] C. T. Zamfirescu, On hypohamiltonian and almost hypohamiltonian graphs, J. Graph Theory 79 (2015), 63-81. 